\title{
ATTENTION DEFICIT DISORDER
}

\section{OROS METHYLPHENIDATE IN ADOLESCENTS WITH ADHD}

The efficacy and tolerability of osmotic-release oral system (OROS) methylphenidate in adolescents with attention deficit hyperactivity disorder (ADHD) were evaluated in a multisite controlled study at the Massachusetts General Hospital, Boston, and other centers in the United States. Of 220 adolescents who entered the study, 177 completed an initial dose titration phase and were randomized to receive 2 weeks' treatment with their individualized, effective and tolerated dosage $(18,36,54$, or $72 \mathrm{mg}$ once daily) or placebo. Investigator, parent and patient report measures showed a significant reduction from baseline in ADHD symptoms in $52 \%$ of subjects treated with OROS compared to $31 \%$ of those receiving placebo. The maximum dose of $72 \mathrm{mg} / \mathrm{d}$ was required to effect significant improvement in $37 \%$ of subjects. Drug-related adverse events included insomnia in $4.6 \%$, headache in $3.4 \%$, decreased appetite (2.3\%), and diarrhea (2.3\%). (Wilens TE, McBurnett K, Bukstein O, et al. Multisite controlled study of OROS methylphenidate in the treatment of adolescents with attention-deficit/hyperactivity disorder. Arch Pediatr Adolesc Med Jan 2006;160:82-90). (Respond: Timothy E Wilens MD, Massachusetts General Hospital, YAW-6-6A, 32 Fruit St, Boston, MA 02114).

COMMENT. OROS methylphenidate is effective in $50 \%$ of adolescents with ADHD following a short-term trial, but larger doses than usual are required in more than one-third of patients. Although side effects were relatively infrequent and mild, the patient population was selected, and included only those previously found to tolerate and respond to individualized doses. Further study is required to demonstrate long-term effectiveness and tolerability at these larger doses.

Response to methylphenidate and familial psychopathology. In a double-blind, placebocontrolled, randomized 2-week crossover trial of low dose MPH $(0.5 \mathrm{mg} / \mathrm{kg} / \mathrm{d}$ in 2 equal doses), the first-degree relatives of good responders (GR) were at significantly higher risk of $\mathrm{ADHD}$, and second-degree relatives of GR were at significantly higher risk of antisocial personality disorder, than relatives of poor responders (PR). Family history of ADHD and psychopathology may help to distinguish ADHD GR from PR. (Grizenko N et al. J Am Acad Child Adolesc Psychiatry Jan 2006;45:47-53).

\section{INFECTIOUS DISORDERS}

\section{LUMBAR PUNCTURE IN LATE ONSET NEONATAL INFECTION}

Five relevant clinical studies (1980-2004) found in a Medline, Pubmed and other database search were analyzed to determine the incidence of meningitis and the importance of routine lumbar puncture (LP) in late onset neonatal infection (greater than 48 hours), in a report from Northwick Park Hospital, Harrow, UK. CSF culture was positive in $1.3-3.5 \%$ of infants with suspected infection. Blood cultures were negative in $15-30 \%$ of infants with 
meningitis and positive CSF culture. Except for one study, LPs were not routine for investigation of infection, and were obtained in only $60 \%$ of those with positive blood culture. Almost one third of neonates with sepsis had coexisting meningitis.

Late onset meningitis is associated with predominantly Gram negative organisms. The incidence of viral and fungal (Candida albicans) organisms is higher in late onset compared to early onset infection. Group B streptococcal infection presenting late is more likely to be associated with meningitis.

Low rates of LP were attributed to perceived adverse effects of the procedure, the babies being considered "too sick to tap." In the studies reviewed, LP was not associated with complications (risk of trauma, infection, spinal epidermoid tumor, brain stem herniation, or hypoxic stress). The risk of death was $23 \%$ in infants with meningitis versus $9 \%$ in those with LP and no meningitis. The rate of positive CSF cultures among patients with LP was not significantly different across centers. The importance of a repeat LP to determine effectiveness of treatment is emphasized by the finding of 10 of 90 repeat cultures being positive for the same organism. (Malbon K, Mohan R, Nicholi R. Should a neonate with possible late onset infection always have a lumbar puncture? Arch Dis Child January 2006;91:75-76). (K Malbon, Neonatal Unit, Northwick Park Hospital, Harrow HA1 3UJ, UK).

COMMENT. The authors conclude that lumbar puncture should be considered as part of the routine investigation of late onset infection (after 48 hours) in neonates. LP performed in 30 to 90 infants (depending on the patient population) during investigation for serious bacterial illness would detect one case of meningitis.

\section{NEUROCUTANEOUS SYNDROMES}

\section{LINEAR SCLERODERMA AND NEUROLOGICAL COMPLICATIONS}

Three patients with linear scleroderma en coup de sabre who presented with neurologic abnormalities before or concurrent with the dermatologic diagnosis are reported from the Medical College of Wisconsin, Milwaukee, WI. Case 1, a 5-year-old girl presented with partial complex seizures and a linear plaque with alopecia from the left eyebrow to the scalp. The EEG showed focal epileptiform discharges in the left posterotemporal area. Despite treatment with topical corticosteroids, the skin lesions first spread to form indurated plaques, and stabilized after 18 months. Seizures responded to antiepileptic drugs (AED), and treatment was tapered after 4 years. The child was seizure-free for 8 years, and AED were restarted at age 14, after a generalized seizure recurrence. Repeat MRIs were normal. Skin lesions consisted of hyperpigmentation and linear depressed plaques over the forehead. Case 2, a 6-year-old boy presented with right facial palsy, and headaches. Annular, erythematous areas of alopecia appeared on the right parietal scalp several months later. MRI was normal. Neurologic and skin lesions stabilized after treatment with prednisolone and methotrexate. Case 3, a 5 year-old girl presented with a white patch in the midline of her forehead, and 2 months later, she had a seizure. Uncontrolled partial complex seizures followed. Video-EEG showed a left frontal-temporal focus, a repeat MRI showed a T2 signal in the left subcortical white matter, CT revealed frontal calcifications and encephalomalacia, and PET scan had hypometabolic areas in the left frontal lobe. Resection of the left frontal area showed 